Artículo original

\title{
Análisis polínico del propóleo rojo de la zona insular de San Andrés, Colombia
}

\section{Palynologycal analysis of red propolis from San Andrés insular zone, Colombia}

\author{
드 Guillermo Salamanca-Grosso, (1) Mónica Patricia Osorio-Tangarife \\ Grupo de Investigaciones Mellitopalinológicas y Propiedades Fisicoquímicas de Alimentos, Departamento de Química, \\ Facultad de Ciencias, Universidad del Tolima, Ibagué, Colombia
}

\begin{abstract}
Resumen
La diversidad de las especies vegetales en los ecosistemas es fundamental para la preservación de las especies de polinizadores y, especialmente, de las abejas, porque dependen directamente del suministro de polen y néctar. El espectro polínico de propóleos es un indicador de la vegetación en zonas biogeográficas determinadas y evidencia, de alguna manera, la relación entre plantas e insectos en la búsqueda de recursos para el sustento de las colonias de abejas. Se evaluaron cinco muestras de propóleo rojo recolectadas en la zona insular de San Andrés, Colombia, para determinar la frecuencia y los tipos polínicos presentes. Las muestras se sometieron a operaciones de separación y concentración y, posteriormente se trató el sedimento mediante acetólisis. Se pudieron reconocer 46 tipos polínicos en 29 familias botánicas, con predominio de Cyperus haematodes, Avicennia germinans, Conocarpus erectus, Dalbergia brownei, Laguncularia racemosa, Rhizophora mangle, Sesuvium portulacastrum, Cocos nucifera y Spondias mombin, y otros tipos menos importantes como Morinda citrifolia, según sus frecuencias polínicas. Las familias con mayor número de tipos polínicos fueron Acanthaceae, Aizoaceae, Arecaceae, Combretaceae y Rhizophoraceae. Estos resultados constituyen uno de los primeros trabajos de análisis polínico en propóleos colombianos.
\end{abstract}

Palabras clave: análisis polínico; origen botánico; propóleo rojo.

\begin{abstract}
The diversity of plant species present in ecosystems is critical to the preservation of pollinator species and especially bees because they depend directly on the supply of pollen and nectar. The pollen spectra of propolis is an indicator of the vegetation existing in certain biogeographic area and it evidences in some way the relation plant-insect in the search for livelihood resources for the bee colonies. We evaluated five red propolis samples collected in San Andres Island (Colombia) to determine the frequency and pollen types present. The samples underwent separation and concentration, as well as sediment analysis by acetolysis. The work allowed the recognition of 46 pollen types from 29 botanical families, predominantly Cyperus haematodes, Avicennia germinans, Conocarpus erectus, Dalbergia brownei, Laguncularia racemosa, Rhizophora mangle, Sesuvium portulacastrum, Cocos nucifera, and Spondias mombin and other less important types, such as Morinda citrifolia, revealed through their pollen frequencies. The families with the highest number of pollen types present corresponded to Acanthaceae, Aizoaceae, Arecaceae, Combretaceae, and Rhizophoraceae. These results represent one of the first pollen analysis in Colombian propolis.
\end{abstract}

Keywords: Pollen analysis; botanical origin; red propolis.

\section{Introducción}

El propóleo es un producto natural de consistencia cauchosa y pegajosa. Es elaborado por las abejas a partir de resinas, gomas y exudaciones de las plantas que han sido modificadas tras la adición de enzimas y secreciones. Presenta tonalidades de color castaño, marrón, pardo, rojizo y verde, $\mathrm{y}$, en algunos casos, negros, según sea el origen botánico y geográfico (Salamanca, 2017). El producto contiene una amplia variedad de metabolitos, principalmente compuestos de estructura fenólica, a los que se les atribuyen diversas propiedades biológicas. Este material ha alcanzado una posición destacada en el mercado de los productos naturales a nivel mundial (Kuropatnicki, et al., 2013; Fabris, et al., 2013; Wagh, 2013; Banskota, et al., 2001).

\footnotetext{
*Correspondencia:

Guillermo Salamanca-Grosso; gsalaman@ut.edu.co

Recibido: 20 de mayo de 2019

Aceptado: 16 de agosto de 2019

Editor: Elizabeth Castañeda
} 
Los propóleos de diferentes entornos biogeográficos del mundo han llamado la atención de muchos investigadores por sus reconocidas propiedades biológicas benéficas que lo proyectan como un producto de interés general debido a su amplio espectro antimicrobiano y antiinflamatorio, sus efectos cicatrizantes, anestésicos y anticariogénicos, su acción estimulante del sistema inmunológico, de protección del hígado, contra el cáncer, antiviral, antifúngica, antiprotozoaria, antirradicalaria, antioxidante y como agente recuperador de tejidos (Salamanca, 2017; Freires, et al., 2016; Silva-Carvalho, et al., 2015; Fabris, et al., 2013; Olivieri, et al., 2013; Sulaiman, et al., 2012; Urushisaki, et al., 2011; Valente, et al., 2011; Li, et al., 2009; Banskota, et al., 2001). El origen botánico de muchos propóleos se ha determinado mediante el análisis polínico y por comparación de fracciones epidérmicas de plantas de referencia con secciones aisladas de propóleos. Con el advenimiento de los métodos instrumentales de análisis se han podido determinar más de 300 principios activos según su origen biogeográfico en zonas templadas y tropicales del mundo (Urushisaki, et al., 2011).

Las propiedades del propóleo dependen de la composición de las plantas visitadas, de las resinas o exudados que las abejas hayan usado para formar el producto, de la forma de recolección, de las condiciones climáticas y del periodo en el cual haya sido beneficiado (Salamanca, 2017; Urushisaki, et al., 2011; Valente, et al., 2011; SilvaCarvalho, et al., 2015; Li, et al., 2009). En general, los propóleos tienen forma diversas: de aspecto duro o quebradizo, de textura suave, flexible o pegajosa, semidura o blanda, según la presencia de bálsamos, cera, gomas, resinas y restos de plantas (Sulaiman, et al., 2012; Freires, et al., 2016; Olivieri, et al., 2013; Salatino, et al., 2011).

A diferencia de las zonas templadas, en ambientes tropicales las abejas acuden a otras fuentes para suplir las necesidades de sostenimiento de las colonias y buscar resinas y exudados de las plantas para la elaboración de sus propóleos (Silva-Carvalho, et al., 2015; Miguel, et al., 2014; Bankova, et al., 2014; Rodríguez, et al., 2012). En consecuencia, se presentan importantes diferencias en su composición química, la cual depende de la flora local (Fabris, et al., 2013).

El análisis polínico, técnica de identificación del origen botánico de los propóleos, ha demostrado gran potencial como herramienta de diagnóstico. Los primeros trabajos de identificación del origen botánico conforme al origen geográfico de los propóleos, se remontan al final de la década de 1970 (D'Albore, 1979; Theobald, et al., 1979; Barth, 1998). Habitualmente, los componentes de las masas mecánicas del propóleo suelen ser filamentos de las hojas de las plantas, fragmentos de tricomas y fracciones de hojas y tallos, que pueden ser orientativos (Theobald, et al., 1979). Con esta técnica se ha identificado la presencia de polen de Eucalyptus sp., Populus sp., Pinus alba, P. nigra y $P$. trémula, (Freires, et al., 2016), de Dalbergia ecastophyllum,
Macaranga tanarius y Cupressus sempervirens, entre otros (Bankova, et al., 2014; Daugsch, et al., 2008; Melliou, et al., 2007).

Entre los trabajos de identificación de fuentes florales propias de los propóleos tropicales de América, se destacan los de Brasil, en donde se ha identificado el espectro polínico de bosques secos de Bahia, Estado de Brasil, y se han descrito algunos tipos polínicos como Cupania, Eucalyptus, Schinus, que son propios de zonas costeras (Oliveira \& Riveiro, 2018; Matos \& Santos, 2016; Matos, et al., 2014); no obstante, en el polen de Río de Janeiro, Rio Grande do Sul y Minas Gerais se han relacionado especies de Eucalyptus, Eupatorium, Cecropia y Mimosa caesalpiniaefolia (Barth, 1998). En este último estado se identificaron granos de polen de Vellozia sp., Baccharis dracunculifolia, Eucalyptus sp., Hyptis sp. y Vernonia polyanthes, (Barth, et al., 1999). Asimismo, se han relacionado diferentes entornos biogeográficos del propóleo rojo. En Cuba estos corresponden a la localidad de Pinar del Río, en México, a Champotón, en el nordeste de Brasil, la zona de manglares, y a la isla de San Andrés en Colombia (Salamanca \& Osorio, 2018; Omar, et al., 2016; Piccinelli, et al., 2011; Lotti, et al., 2010).

Los propóleos de tonalidades rojizas de Cuba y México se han relacionado con especies del género Clusia $(C$. grandiflora, C. lanceolata, C. paralicola, C. parviflora y C. espi-sanctensis) (Corbellini, et al., 2017). Los de Brasil han revelado la presencia de granos de polen de Schinus, Protium, Rhizophora, Laguncularia, Avicennia y D. ecastophyllum (Barth \& Luz, 2009), en tanto que en el polen verde o marrón de Río de Janeiro, Rio Grande do Sul y Minas Gerais se han relacionado especies de Eucalyptus, Eupatorium, Mimosa caesalpiniaefolia y Cecropia (Barth, 1998). En esta última región se identificaron granos de polen de Vellozia sp., B. dracunculifolia, Eucalyptus sp., Hyptis sp. y $V$. polyanthes (Barth, et al., 1999) ; en Sao Paulo, se han identificado hasta 28 tipos polínicos, principalmente de las familias Leguminosae, Mimosoideae y Myrtaceae, (Silva, et al., 2013), y en geopropóleos los componentes mayoritarios se relacionan con el sílice, la arcilla y la ausencia de tricomas (Barth, 2006; Barth \& Luz, 2003; Absy, et al., 1984; Absy \& Kerr, 1977).

La flora apícola de mayor interés involucra una serie de plantas propias que se pueden clasificar según las zonas de vida y los pisos térmicos, como lo indican varios trabajos de identificación taxonómica y palinológica de plantas visitadas por las abejas en áreas andinas y páramos, en el Caribe, o consociaciones geográficas de ultramar, como en el caso de la isla de San Andrés (García, et al., 2011; Velandia, et al., 2012; Velásquez \& Rangel, 1995). Asimismo, en el sureste de Brasil se han analizado sedimentos de propóleos y geopropóleos y se ha determinado que en los verdes, rojos y marrones sobresalen las especies de asteraceas y eucaliptos, mientras que entre los geopropóleos predominan las de Cecropia y Melastomataceae (Barth \& Freitas, 2015). Cabe destacar que en estudios de la Universidad Nacional de 
Colombia se ha identificado la flora vascular terrestre de San Andrés (Lowy-Cerón, 2000), pero no hay reporte alguno sobre la identificación palinológica en el Archipiélago. Los trabajos polínicos revisten importancia taxonómica y contribuyen a dilucidar la relación entre plantas e insectos en los ecosistemas, cuyo estudio es determinante para identificar el origen botánico de la miel y los propóleos. Dichos estudios, sin embargo, son limitados y las publicaciones sobre el tema no son abundantes. Entre estas se destacan las investigaciones en muestras de propóleo de Turquia, Portugal y Brasil.

El polen es un componente habitual en muestras de propóleo; este proviene de las anteras de las flores visitadas por las abejas y es un indicador del origen botánico. En Colombia la cosecha y el beneficio de propóleos han permitido identificar los tipos de las muestras en función de las zonas de vida (Salamanca, 2017). Las determinaciones polínicas en esta matriz aún no se han relacionado ni se han considerado los aspectos polínicos como parámetro de autenticidad del producto. El análisis polínico en muestras de propóleo colombiano no se ha hecho y los reportes de los datos obtenidos en otros países (Corbellini, et al., 2017; Barth \& Luz, 2009) solo proporcionan una referencia para iniciar los estudios en Colombia. En este contexto el presente trabajo se propuso estudiar los sedimentos polínicos de muestras de propóleo rojo de la isla de San Andrés después de una fase de extracción de grasas y resinas para enriquecer la naturaleza del sedimento.

\section{Materiales y métodos}

Zona de estudio. El entorno biogeográfico donde se recolectaron las muestras correspondió a la isla de San Andrés $\left(12^{\circ}\right.$ $35^{\prime} 37^{\prime}$ y $14^{\circ} 42^{\prime} \mathrm{N}$ y $81^{\circ} 40^{\prime} 49^{\prime \prime}$ y $81^{\circ} 43^{\prime} 13$ ' O). La Isla posee una altitud de $85 \mathrm{~m}$ s.n.m. y la temperatura media es de $27,4{ }^{\circ} \mathrm{C}$ (Figura 1); predomina un sistema de precipitación bimodal: 121 a $307 \mathrm{~mm} / \mathrm{mes}$ en el periodo lluvioso y 23 a 94 en el periodo seco. La humedad relativa multianual es del $82 \%$. Los ecosistemas de manglares son típicos y poseen reductos de flora endémica, propia de sistemas insulares caribeños. La flora predominante se caracteriza por elementos de bosque seco (bs-T), principalmente, con algunas especies presentes en la consociacion de bosque húmedo tropical (bh-T), (Valle, et al., 2011; Uribe \& Urrego, 2009). El relieve de la isla está formado por una cadena de colinas que se extiende a lo largo de ella en su parte central, bordeada por una morfología plana de plataforma arrecifal emergida. Estas colinas constituyen un sistema montañoso longitudinal que se inicia al NE y se prolonga hacia el sur, alcanzando alturas máximas de $109 \mathrm{~m}$.

Material biológico. Se recolectaron cinco muestras de propóleo rojo de colmenas Langstroth establecidas en cuatro apiarios cercanos a la zona de manglar de Bahia Hooker (BH: $12^{\circ} 56^{\prime} 58^{\prime \prime} \mathrm{N}$ y $81^{\circ} 70^{\prime} 62^{\prime \prime} \mathrm{O}$ ) y El Cove (EC: $12^{\circ} 54^{\prime} 35^{\prime}$ 'N y 81 $\left.72^{\prime} 62^{\prime \prime} \mathrm{O}\right)$ y Flowers Hill (FH: 12 $19^{\prime} 7.2^{\prime \prime} \mathrm{N}$ y $81^{\circ} 42$ '46"O), entre febrero y junio de 2016, mediante operaciones de raspado de los cuadros de las colmenas de abejas Apis mellifera L. (Hymenoptera: Apidae) de linaje europeo tipo M (Salamanca, et al., 2014). El material recolectado se dispuso en recipientes de vidrio de color ámbar con tapa de desenroscar y se refrigeraron a $4{ }^{\circ} \mathrm{C}$, para posteriormente trasladarlos a la Universidad del Tolima, donde se congelaron a $-20^{\circ} \mathrm{C}$, y completar las evaluaciones.

Caracterizaciones polínicas. Las muestras se procesaron usando una nueva metodología basada en una limpieza total, incluida la eliminación de ceras y resinas contenidas en los propóleos mediante la extracción Soxleth. En este caso, $1 \mathrm{~g}$ de propóleo crudo fue lixiviado con éter de petróleo para eliminar ceras y grasas con etanol al $96 \%$ para remover gomas y resinas. Una vez hecha la limpieza total, el sedimento obtenido se sometió a acetólisis usando la técnica de Erdtman (1969). Una vez se cumplió con el protocolo, los sedimientos procesados se dispusieron en glicerina en tubos Eppendorf. Para su reconocimiento e identificación, las muestras se colocaron en un portaobjetos y se les agregó gelatina glicerinada para fijarlas $\mathrm{y}$ observarlas en el microscopio binocular $\left(\mathrm{AmScope}^{\mathrm{TM}}\right)$.

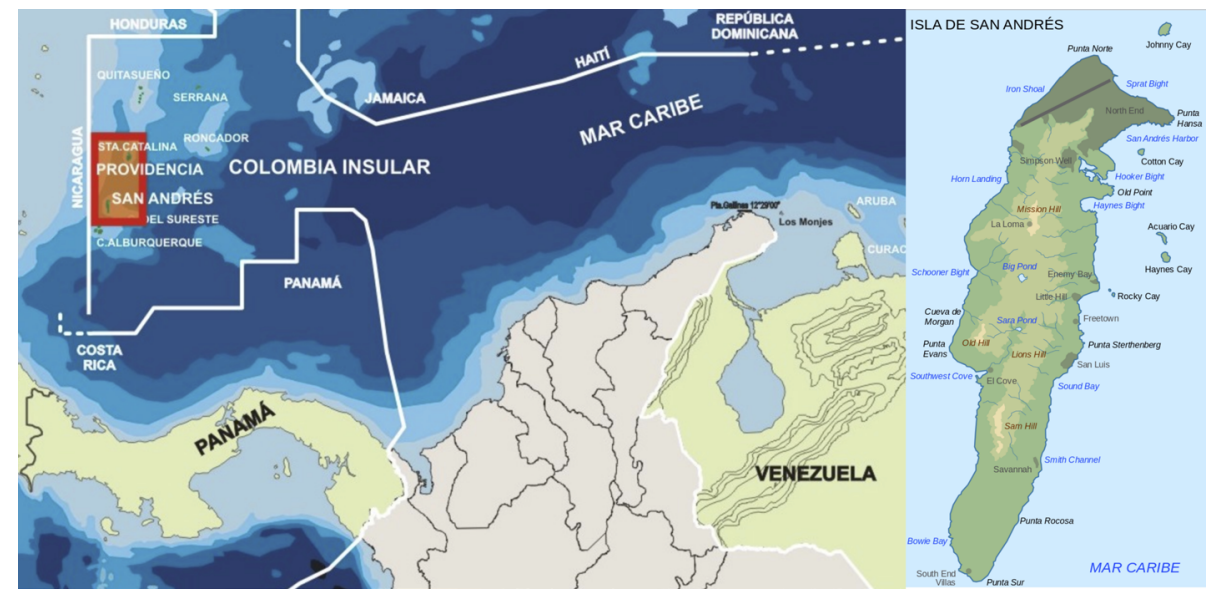

Figura 1. Posición geográfica de la isla de San Andrés, en zona insular colombiana del mar Caribe 
Los registros polínicos se tomaron en una cámara digital $\left(\right.$ Moticam $^{\mathrm{TM}}$ ), con el programa Motic Image Plus ${ }^{\mathrm{TM}} 2.0 \mathrm{ML}$ acoplado al microscopio. En las evaluaciones del sedimento se hicieron barridos sobre las preparaciones acetolizadas que condujeron a la captura de imágenes a $10 \mathrm{X}, 40 \mathrm{X}$ y $100 \mathrm{X}$. Más de 350 granos de polen de cada muestra se contaron directamente bajo el microscopio, además, se determinaron las frecuencias absolutas totales y la frecuencia relativa en el grupo de muestras estudiado. La identificación polínica se hizo haciendo uso de atlas polínicos de referencia de la costa caribe colombiana (García, et al., 2011; Willard, et al., 2004), y de manglares del noreste brasilero (Oliveira \& Ribeiro, 2018; Matos \& dos Santos, 2016; Freitas, et al., 2013; Magalhães \& Ribeiro, 2009) que se compararon con otros trabajos de palinología en propóleos (Barth \& Freitas, 2015).

Estadística. Se determinaron los conteos superiores a 400 granos de polen en cada uno de los sedimentos de las cinco muestras de propóleo rojo recolectadas, con los cuales se estimó la riqueza polínica por muestra y la frecuencia relativa con respecto a los granos. Además, se proyectó el análisis de conglomerados en un gráfico de constelación en función de los tipos polínicos dominantes. Estas evaluaciones se hicieron con el paquete estadístico JMP ${ }^{\circledR}$ Pro 14.0.0.

\section{Resultados}

En general, los tipos polínicos hallados en las muestras de los sedimentos de propóleo rojo correspondieron a 46 especies botánicas de interés apícola en las cinco zonas estudiadas (FH, ECi, ECii, BHi y BHii), entre las cuales predominaron C. nucifera, A. germinans, L. racemosa, $C$. erectus, $R$. mangle y $S$. mombin, que representaron el $13 \%$ de los tipos polínicos identificados. La mayor frecuencia relativa en el total de tipos polínicos contados (2.616) de este grupo se presentó en $C$. nucifera $(10,4 \%)$, seguida de la de especies de manglar tales como A. germinans $(8,4 \%)$, y $L$. racemosa y $R$. mangle, cada una con $7,8 \%$. Por su parte, $S$. portulacastrum, especie de interés apícola, se halló recurrentemente en los sedimientos de todas las muestras, con una frecuencia relativa de 5,3\%; otras especies encontradas fueron C. haematodes $(3,7 \%)$, Artocarpus altilis y Psidium guajava (2,9\% cada una), $M$. citrifolia $(2,8 \%)$ y Crotalaria retusa $(0,5 \%)$. Las familias con mayor número de polen fueron Combretaceae (16,8 \%), Acanthaceae y Arecaceae (10,4 \%) y Rhizophoraceae (7,8 \%). Después de la extracción Soxtleh persistieron pequeños fragmentos redondeados de tonos marrón oscuro y rojizo que se observaron incluso después, y que son atribuibles a la elevada fracción de células resinosas. Los tricomas no son abundantes y no se identificaron hifas o esporas fúngicas. El análisis de conglomerados proyectado como gráfico de constelación, reveló cinco grupos específicos (Figura 2) que representan la riqueza polínica de las muestras estudiadas. En total, 29 familias botánicas se han relacionado a partir de la identificación polínica en los sedimentos de las muestras. El 2,5\% de los granos polínicos no fueron asignados a ningún grupo, ya que no se logró su identificación. La Tabla 1 recoge los tipos de polen clasificados según la riqueza polínica y la frecuencia relativa, los cuales se relacionan con la representación del análisis de conglomerados de la Figura 2.

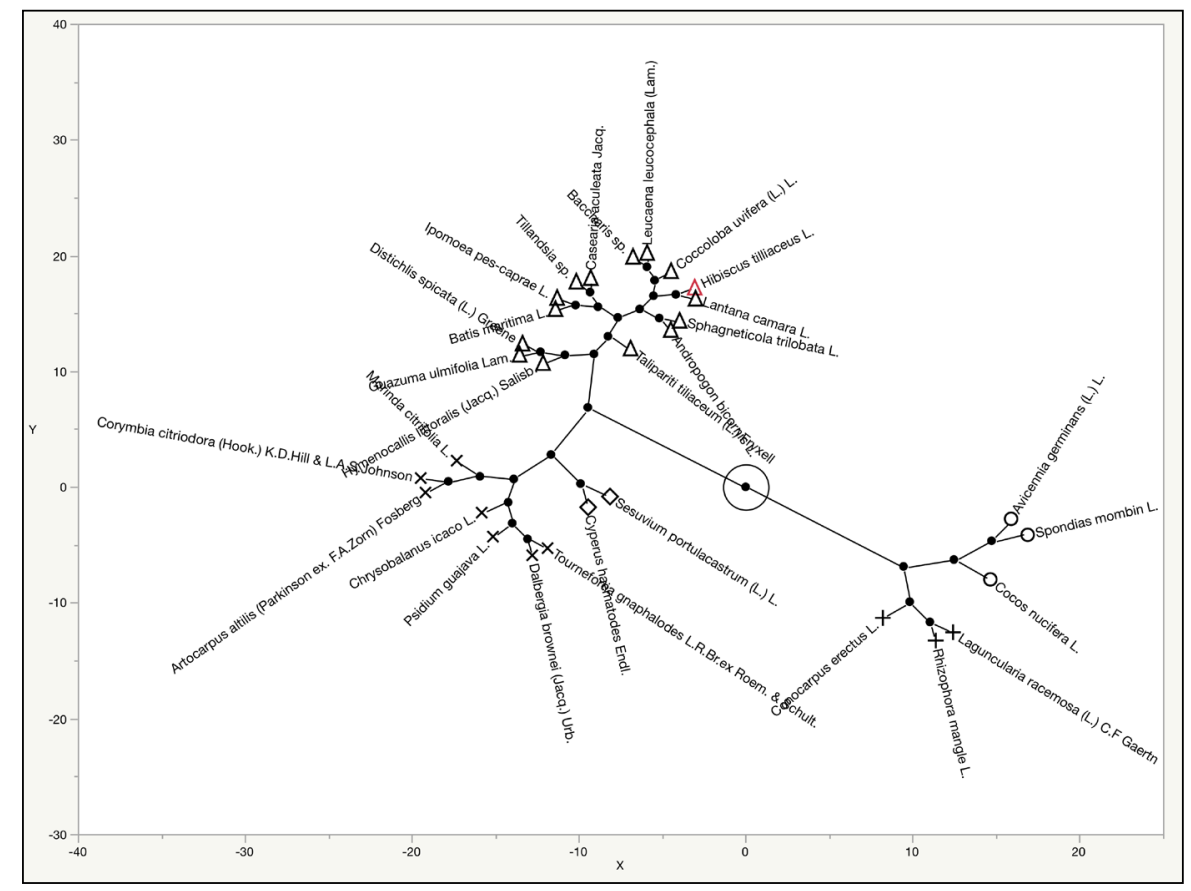

Figura 2. Análisis de conglomerados presentado como estructura de constelación de los tipos polínicos identificados en los sedimentos de propóleo rojo de la isla de San Andrés, Colombia 
Tabla 1. Conteo de tipos polínicos identificados en las muestras de propóleo rojo recolectadas en la isla de San Andrés, Colombia

\begin{tabular}{|c|c|c|c|c|c|c|c|}
\hline Familia & Tipos polínicos & BHi & FH & BHii & $\mathbf{E C i}$ & ECii & FR \\
\hline Amaranthaceae: & Amaranthus sp. & & 9 & 3 & 7 & 0 & 0,7 \\
\hline \multirow[t]{2}{*}{ Acanthaceae: } & Avicennia germinans (L.) L. & 47 & 48 & 52 & 38 & 67 & 9,6 \\
\hline & Ruellia tuberosa $\mathrm{L}$. & & 3 & 3 & 7 & 7 & 0,8 \\
\hline Aizoaceae: & Sesuvium portulacastrum (L.) L. & 16 & 30 & 34 & 33 & 26 & 5,3 \\
\hline Amaryllidaceae: & Hymenocallis littoralis (Jacq.) Salisb. & 3 & 7 & 3 & 12 & 11 & 1,4 \\
\hline \multirow[t]{3}{*}{ Anacardiaceae: } & Schinus terebinthifolia Raddi. & & 0 & 2 & 0 & 1 & 0,1 \\
\hline & Spondias mombin L. & 41 & 48 & 38 & 36 & 39 & 7,7 \\
\hline & Tapirira sp. & & 1 & 6 & 7 & 4 & 0,7 \\
\hline Annonaceae: & Annona glabra L. & & 3 & 0 & 8 & 3 & 0,5 \\
\hline Apiaceae: & Coriandrum sativum $\mathrm{L}$. & & 6 & 5 & 3 & 1 & 0,6 \\
\hline Arecaceae: & Cocos nucifera $\mathrm{L}$. & 47 & 39 & 82 & 50 & 55 & 10,4 \\
\hline Bataceae: & Batis maritima $\mathrm{L}$. & 6 & 7 & 3 & 4 & 2 & 0,8 \\
\hline Boraginaceae: & Tournefortia gnaphalodes L.R.Br.ex Roem. \& Schult. & 14 & 7 & 19 & 14 & 11 & 2,5 \\
\hline Bromeliaceae: & Tillandsia sp. & 6 & 4 & 3 & 2 & 1 & 0,6 \\
\hline \multirow[t]{3}{*}{ Combretaceae: } & Terminalia catappa $\mathrm{L}$. & & 5 & 2 & 4 & 5 & 0,6 \\
\hline & Laguncularia racemosa (L.) C.F Gaertn & 30 & 37 & 45 & 50 & 58 & 8,4 \\
\hline & Conocarpus erectus $\mathrm{L}$. & 22 & 29 & 52 & 39 & 61 & 7,8 \\
\hline \multirow[t]{4}{*}{ Asteraceae: } & Acmella darwinii (D.M.Porter) R.K.Jansen & & 2 & 1 & 1 & 3 & 0,3 \\
\hline & Baccharis sp. & 4 & 2 & 3 & 4 & 4 & 0,6 \\
\hline & Bidens pilosa $\mathrm{L}$. & & 5 & 1 & 7 & 4 & 0,6 \\
\hline & Sphagneticola trilobata $\mathrm{L}$. & 7 & 2 & 1 & 1 & 6 & 0,6 \\
\hline Convovulaceae: & Ipomoea pes-caprae L. & 5 & 6 & 3 & 2 & 6 & 0,8 \\
\hline Cyperaceae: & Cyperus haematodes Endl. & 11 & 23 & 31 & 12 & 21 & 3,7 \\
\hline Chrysobalanaceae: & Chrysobalanus icaco L. & 4 & 11 & 21 & 9 & 8 & 2,0 \\
\hline Euphorbiaceae: & Hippomane mancinella $\mathrm{L}$. & & 9 & 0 & 3 & 0 & 0,5 \\
\hline \multirow[t]{4}{*}{ Leguminosae: } & Crotalaria retusa $\mathrm{L}$. & & 0 & 7 & 2 & 3 & 0,5 \\
\hline & Dalbergia brownei (Jacq.) Urb. & 16 & 12 & 13 & 11 & 8 & 2,3 \\
\hline & Leucaena leucocephala (Lam.) & 5 & 0 & 3 & 3 & 4 & 0,6 \\
\hline & Mimosa tenuiflora (Willd.) Poir & & 2 & 0 & 7 & 2 & 0,4 \\
\hline \multirow[t]{4}{*}{ Malvaceae: } & Guazuma ulmifolia Lam. & 4 & 5 & 6 & 7 & 7 & 1,1 \\
\hline & Hibiscus tilliaceus $\mathrm{L}$. & 2 & 3 & 2 & 5 & 3 & 0,6 \\
\hline & Talipariti tiliaceum (L.) Fryxell & 8 & 12 & 4 & 2 & 0 & 1,0 \\
\hline & Triumfetta lappula $\mathrm{L}$. & & 1 & 2 & 1 & 2 & 0,2 \\
\hline Moraceae: & Artocarpus altilis (Parkinson ex. F.A.Zorn) Fosberg & 17 & 3 & 21 & 13 & 22 & 2,9 \\
\hline \multirow[t]{2}{*}{ Myrtaceae: } & Psidium guajava $\mathrm{L}$. & 11 & 14 & 17 & 21 & 13 & 2,9 \\
\hline & Corymbia citriodora (Hook.) & 13 & 7 & 6 & 11 & 21 & 2,2 \\
\hline \multirow[t]{2}{*}{ Poaceae: } & Andropogon bicornis L. & 7 & 3 & 4 & 4 & 7 & 1,0 \\
\hline & Distichlis spicata (L.) Greene & 3 & 4 & 7 & 3 & 9 & 1,0 \\
\hline Polygonaceae: & Coccoloba uvifera $\mathrm{L}$. & 3 & 0 & 3 & 2 & 7 & 0,6 \\
\hline Rhizophoraceae: & Rhizophora mangle $\mathrm{L}$. & 33 & 33 & 41 & 52 & 44 & 7,8 \\
\hline Rosaceae: & Rubus urticifolius Poir. & & 1 & 0 & 3 & 0 & 0,2 \\
\hline Rubiaceae: & Morinda citrifolia $\mathrm{L}$. & 23 & 16 & 12 & 8 & 14 & 2,8 \\
\hline Salicaceae: & Casearia aculeata Jacq. & 5 & 3 & 6 & 3 & 0 & 0,6 \\
\hline Sapindaceae: & Cupania glabra Sw. & & 2 & 5 & 1 & 3 & 0,4 \\
\hline Verbenaceae: & Lantana camara L. & 5 & 3 & 0 & 5 & 3 & 0,6 \\
\hline \multirow[t]{3}{*}{ No identificados } & No identificados & & 11 & 21 & 15 & 19 & 2,5 \\
\hline & Total de granos polínicos contados & 418 & 478 & 593 & 532 & 595 & 2616 \\
\hline & Total de tipos de polen identificados & 30 & 46 & 46 & 46 & 46 & \\
\hline
\end{tabular}

BH: Bahia Hooker. EC: El Cove. FH: Flowers Hill. FR: Frecuencia relativa total 
El análisis de la distribución de frecuencias predominantes de los tipos polínicos (Figura 3) permitió identificar dos grupos característicos preponderantes: A. germinans, $C$. nucifera y $S$. mombin fueron las especies más predominantes en los sedimentos según los porcentajes de frecuencia (entre 8,0 y 16,0\%); el segundo grupo mayoritario incluyó dos subgrupos en los que las especies $C$. erectus, $C$. haematodes, L. racemosa y $R$. mangle predominaron. En la Figura 4, se presentan algunas microfotografías de tipos polínicos observados en el microsopio de luz, característicos del sedimento de los propóleos rojos de la zona de la isla de San Andrés (Colombia).

\section{Discusión}

En términos generales, la estructura boscosa de la isla de San Andrés en la Bahía Hooker y El Cove tienen un alto grado de desarrollo y se ubican detrás de la barrera arenosa, lo cual se debe a que permanecen inundados gran parte del año, bien sea por el sistema de lluvias o por el aporte de los drenajes que se depositan sobre los manglares. La unidad El Cove se extiende en un espacio de 1,2 ha y tiene una barrera monoespecífica de $R$. mangle, en tanto que en la Bahía Hooker, que se extiende a lo largo de 34 ha, hay 15,1 ha de $R$. mangle, 0,97 ha de $A$. germinans y 18,4 ha de bosques mixtos de $R$. mangle, $L$. racemosa, A. germinans y C. erectus. Los árboles de porte medio alcanzan hasta 5,6 m, con densidades de hasta 119 árboles por hectárea. En este entorno, la biomasa estimada de $R$. mangle es de $14,8 \mathrm{tn} /$ ha/año, la de $A$. germinans es ligeramente mayor, con 13,5 tn/ha/año, y la de L. racemosa es de 8,32. Los manglares de San Andrés son altamente productivos, en comparación con otros sistemas de manglar en el Caribe. La diversidad polínica observada en las muestras de propóleo rojo podría explicarse en función de la diversidad de la flora asociada con la zona de manglares (García-Hansen \& GaviríaChiquazuque, 1996).

Dado que se han detectado solamente seis zonas geográficas en que se producen los propóleos rojos en el mundo, entre ellas las de Colombia (Salamanca \& Osorio, 2018), solo se conocen los trabajos de caracterización palinológica del nordeste de Brasil (Barth \& Luz, 2009; Avelino \& Ribeiro, 2018). Allí hay una amplia biodiversidad florística que permite un mayor espectro polínico y reduce la posibilidad de hacer comparaciones directas con la isla de San Andrés. La flora insular del Caribe presenta algunas diferencias con respecto a las especies del nordeste brasileño, pero en las dos localidades predominan condiciones de manglar y tipos de flora similares, lo que

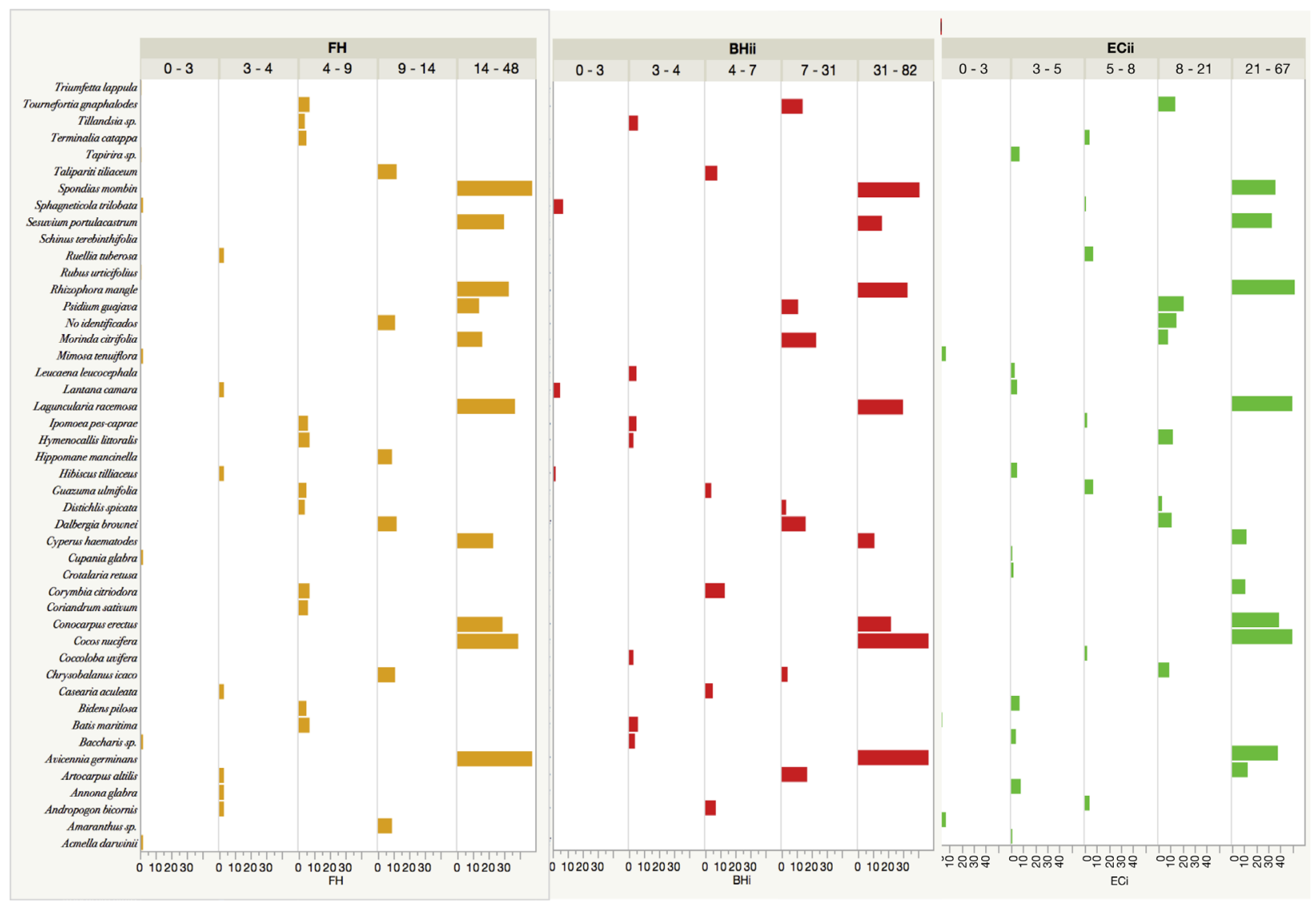

Figura 3. Diagramas característicos de las frecuencias y los tipos polínicos presentes en las muestras de sedimentos de propóleo rojo de las zonas estudiadas en la isla de San Andrés, Colombia. FH, BH y EC: zonas de muestreo. 


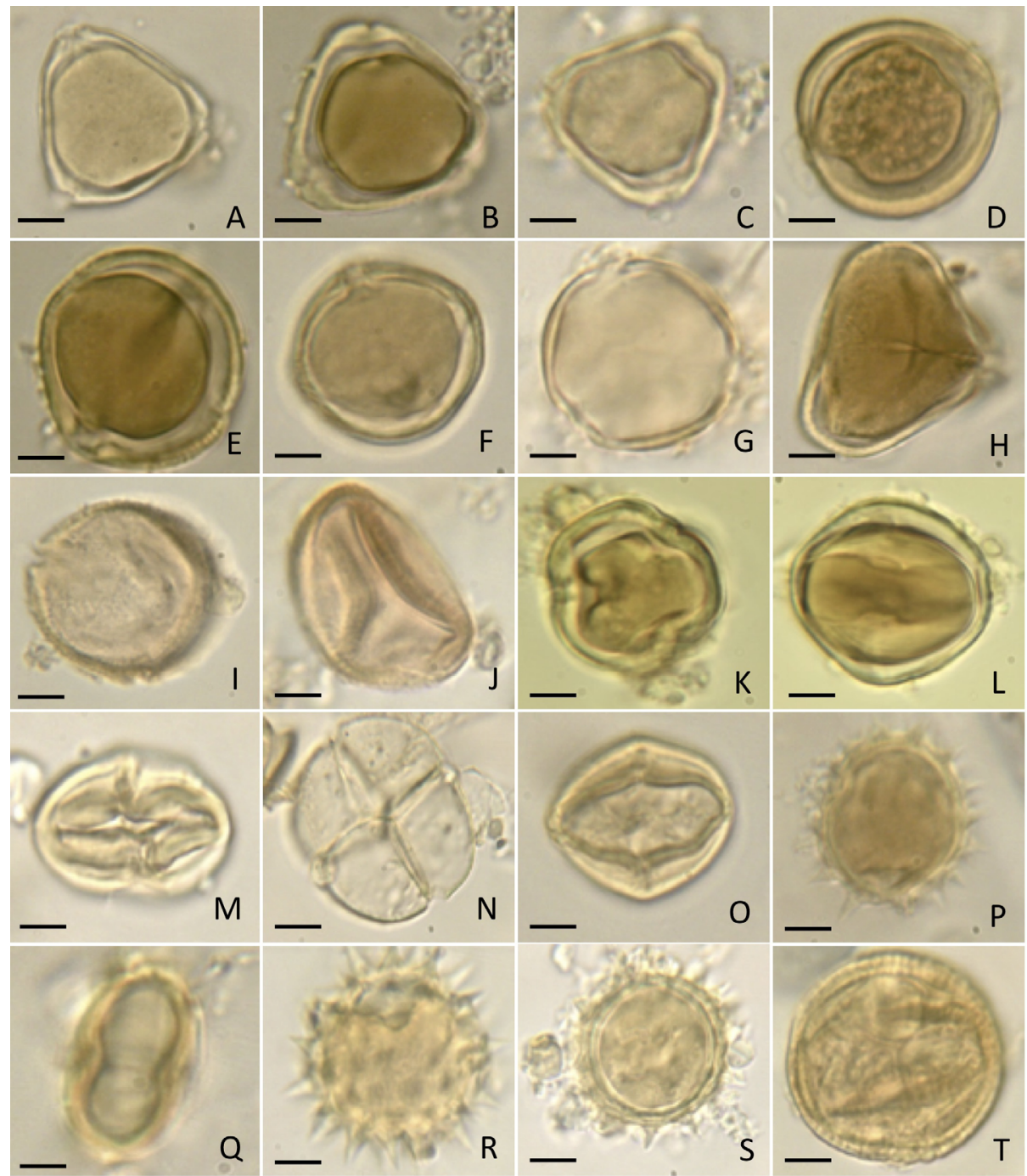

Figura 4. Algunos granos de polen identificados en las muestras de propóleo rojo de la isla de San Andrés, Colombia. A-C. Corymbia citriodora. D-F. Laguncularia racemosa. G. Tapirira sp. H. Cupania glabra I. Spondias mombin. J. Morinda citrifolia. K. Rubus urticifolius. L. Schinus terebinthifolia. M. Triumfetta lappula. N. Mimosa tenuiflora. O. Rhizophora mangle. P. Baccharis sp. Q. Coriandrum sativum. R. Acmella darwinii. S. Bidens pilosa. T. Avicennia germinans. Escala: $10 \mu \mathrm{m}$

explica la presencia de tipos polínicos de Arecaceae $(C$. nucifera), Anacardiaceae (Tapirira $s p$. junto a $S$. mombin), Aizoaceae (S. portulacastrum), Cyperaceae (C. haematodes), Moraceae (A. altilis) y Myrtaceae ( $C$. citriodora), considerados como dominantes, con una frecuencia relativa total de $23,1 \%$, comparable con los conteos de polen de algunas de las muestras recolectadas en el nordeste de Brasil.

El origen de los propóleos rojos del nordeste de Brasil reside principalmente en las resinas de especies de Anacardiaceae y de los géneros Schinus (Barth \& Luz, 2009) y Spondias y Tapiaria (Avelino \& Ribeiro, 2018). En San Andrés, las fuentes de propóleo rojo pueden atribuirse a las exudaciones y resinas de las especies de manglar. En este sentido, es importante resaltar que las fuentes potenciales de propóleo rojo se distribuyen a lo largo del costado oriental de la isla, donde las condiciones hidrológicas, de sedimentación, geomorfológicas y de intensidad del oleaje son favorables para su distribución y predomina la vegetación costera y de manglar $(R$. mangle, asociada con otras especies también presentes: $A$. germinans, $C$. erectus y $L$. racemosa). Además, entre los sectores (Orange Hill y Little Hill), se observan zonas mixtas de R. mangle, L. racemosa, y parches de C. erectus en Smith Channel, Tom Hooker y Savanna, donde se mantiene la unidad del bosque de manglar más desarrollado de la isla.

Los estudios de flora juegan un papel singular cuando se trata del establecimiento de las relaciones entre plantas e insectos. En las muestras de propóleos rojos de Cuba, México, Nigeria y Shandong en China (Corbellini, et al., 2017), no se han hecho evaluaciones polínicas para identificar el origen botánico. Solo en Brasil se han identificado los tipos polínicos que se relacionan con especies de los 
géneros Avicennia, Baccharis, Clusia, Dalbergia, Protium, Rhizophora y Schinus (Barth \& Luz, 2009). En el presente estudio, los tipos polínicos se relacionaron estrechamente con los descritos por Barth \& Luz (2009), con excepción de B. dracunculifolia y Protium, principalmente.

Los tipos polínicos observados correspondieron a plantas de la zona de manglar presentes en el núcleo de la reserva de biosfera "Seaflower" en el archipiélago de San Andrés. El área de estudio no presentaba reductos de vegetación primaria, por el contrario, se evidenciaron formaciones de bosque secundario, con afectación permanente en términos de presión antrópica. En los recorridos realizados por las zonas de manglar siguiendo protocolos de evaluación rápida, se observaron abejas recolectando resinas de los fustes de los árboles e intenso pecoreo sobre las flores y glándulas secretoras de las plantas. En los sedimentos de propóleo rojo observados bajo el microscopio después de la extracción Soxleth y la acetólisis, se evidenciaron fragmentos de tejido vegetal, gomas, ceras, tricomas y abundantes piezas leñosas con residuos de hojas y cortezas, por ejemplo, secciones de neumatóforos.

La asociación vegetal característica que representa el espectro polínico en cada una de las cinco muestras de propóleo rojo estudiadas correspondió a los tipos de mangle identificados como blanco (L. racemosa), negro ( $A$. germinans), botón (C. erectus) y rojo ( $R$. mangle). Se sabe que la especie $S$. portulacastrum es una hierba suculenta que se expande en las zonas costeras y marítimas y que es representativa de la isla de San Andrés, al igual que $C$. nucifera, la más abundante en todos los sedimentos de propóleo rojo analizados. S. mombin, especie que también se destacó en las muestras estudiadas, es característica del Trópico y se extiende a lo largo de las zonas cálidas del país.

Los espectros polínicos caracterizados en este estudio pueden usarse para futuros análisis palinológicos de propóleos rojos, no solo de la isla de San Andrés, sino de otras regiones insulares del mundo aún no consideradas. Por las comparaciones visuales de los diferentes tipos polínicos encontrados en el estudio, así como la remoción de ceras y grasas mediante extracción Soxleth para visualizar mejor los granos, y la acetólisis, el presente documento sirve como punto de partida para posteriores investigaciones. Las especies de mangle registradas son tipos polínicos que dan cuenta de la versatilidad de $A$. mellifera en la isla a la hora de buscar materiales para la protección y sustento de las colonias. El estudio, asimismo, aporta una ruta apropiada para la identificación de polen a partir de matrices complejas, como es el caso de los propóleos.

\section{Conclusiones}

Se evaluaron sedimentos polínicos de muestras de propóleo rojo colombiano de la zona insular de San Andrés, Colombia. El espectro polínico de las muestras presentó algunas diferencias importantes con el del nordeste brasileño. En los sedimentos predominaron tipos polínicos propios de zonas de manglar, además de taxones de las familias Aizoaceae, Arecaceae, Anacardiaceae, Cyperaceae y Myrtaceae, que son representativas de plantas apícolas de las zonas donde se generó el muestreo. Las frecuencias polínicas revelaron grupos predominantes de especies de mangle: $L$. racemos, A. germinans, $C$. erectus y $R$. mangle, así como de $S$. portulacastrum, C. nucifera y $S$. mombin. Además, se observaron tipos polínicos acompañantes y accesorios con bajas frecuencias, que revelan la actividad de $A$. mellifera en la búsqueda de recursos poliníferos para su sustento. El estudio permitió implementar una metodología de tipos polínicos de referencia para establecer el origen botánico de los propóleos.

\section{Contribución de los autores}

GSG: implementación metodológica del proceso de separación y caracterización de matrices de polen en muestras de propóleo, evaluación y análisis de resultados, revisión bibliográfica, y planeación y redacción del manuscrito. MPOT: recepción y procesamiento de las muestras; montaje y caracterización de los tipos polínicos, revisión de la literatura y ajuste y redacción del documento final.

\section{Conflicto de intereses}

Los autores declaran no tener ningún conflicto de intereses con respecto al trabajo realizado. El apoyo financiero para la investigación se reconoció apropiadamente.

\section{Agradecimientos}

Los autores agradecen al grupo de Investigaciones Mellitopalinológicas y Propiedades Fisicoquímicas de Alimentos, a la coordinación General del Proyecto "EOCYT" y a la Oficina de Investigaciones y Desarrollo Científico de la Universidad del Tolima por la financiación del proyecto 410120516. Al señor Cesar Palacio Santos, apicultor de San Andrés, Colombia, por el acompañamiento en el proceso de muestreo. A los pares y evaluadores, por las observaciones y sugerencias durante la fase de evaluación.

\section{Referencias}

Absy, M.L., Camargo, J.M.F., Kerr, W.E., Miranda, I.P.A. (1984). Espécies de plantas visitadas por Meliponinae (Hymenoptera: Apoidea), para coleta de pólen na região do Médio Amazonas. Revista Brasileira de Biologia. 44: 227-237.

Absy, M.L., \& Kerr, W.E. (1977). Algumas plantas visitadas para obtenção de pólen por operárias de Melipona seminigra merrillae em Manaus. Acta Amazônica. 7: 309-315.

Bankova, V., Popova, M., Trusheva, B. (2014). Propolis volatile compounds: Chemical diversity and biological activity: A review. Chemistry Central Journal. 8: 28.

Banskota, A. H., Tezuka, Y., Kadota, S. (2001). Recent progress in Pharmacological Research un Propolis. Phytotherapy Research. 15 (7): 561-571.

Bart., H, O. M., Dutra, V. M. L., Justo, R. L. (1999). Análise polínica de algumas amostras de própolis do Brasil Meridional. Ciência Rural. 29 (4): 663-667. 
Barth, O.M. \& Freitas, S.A. (2015). Palynology as a tool to distinguish between propolis and geopropolis: Southern Brazilian samples. Open Access Library Journal. 2: 2217.

Barth, O. M. (2006). Palynological analysis of geopropolis samples obtained from six species of Meliponinae in the Campus of the Universidade de Ribeirão Preto, USP, Brazil. Apiacta. 41 (2): 71-85.

Barth, O.M. \& Luz, C.F.P. (2003). Análisis palinológico de sedimentos de geopropolis brasileñas. Grana. 42: 121-127.

Barth, O.M. \& Luz, C.F.P. (2009). Palynological analysis of Brazilian red propolis samples. Journal of Apicultural Research and Bee World. 48 (3): 181-188.

Barth, O. M. (1998). Pollen analysis of Brazilian propolis. Grana. 37 (2): 97-101.

Bastos, E.M., Oliveira, V.D.C., Soares, A.E. (2000). Microscopic characterization of the green propolis, produced in Minas Gerais State, Brazil. Honeybee Science. 21: 179-180.

Campos-Trujillo, A., Aguirre-Prieto, A., Muñoz-Romero, G., Rodríguez-Villa, M., Quintana-Martínez, G. (2015). Estudio palinológico de la flora urbana de la ciudad de Chihuahua, México. Acta Botánica Mexicana. 113: 111-134.

Corbellini, L., dos Santos, D., Marinho, F., Pêgas, J.A., Roesch, M., Moura, S. (2017). Red propolis: chemical composition and pharmacological activity. Asian Pacific Journal of Tropical Biomedicine. 7 (7): 591-598.

D'Albore, G. (1979). L'Origine geographique de la propolis. Apidologie. 10: 241-267.

da Silva, C. R., Putarov, T., Oliveira Orsi, R. (2013). Pollen spectrum of propolis samples from São Paulo State, Brazil. Acta Scientiarum, Animal Sciences, Maringá. 35: 297-300.

Daugsch, A., Moraes, C., Fort, P., Park, Y. (2008). Brazilian Red Propolis - Chemical composition and Botanical Origin. Evidence-Based Complementary and Alternative Medicine. 5 (4): 435-441.

Erdtman, G. (1969). An introduction to the study of pollen grains and spores. Editorial Hafner. Scandinavian University Books.

Fabris, S., Bertelle, M., Astafyeva, O., Gregoris, E., Zangrando, R., Gambaro, A., Pereira, G., Stevanato, R. (2013). Antioxidant properties and chemical composition relationship of Europeans and Brazilians Propolis. Pharmacology \& Pharmacy. 4: 46-51.

Freitas, A., Vit, P., Barth, M. (2013). Pollen analysis of geopropolis and propolis from stingless bees. En: Vit, P., \& Roubik, D.W., eds. Stingless bees process honey and pollen in cerumen pots. p. 1-7. Facultad de Farmacia y Bioanálisis, Universidad de Los Andes; Mérida, Venezuela.

Freires, I.A., de Alencar, S.M., Rosalen, P.L (2016). A pharmacological perspective on the use of Brazilian Red Propolis and its isolated compounds against human diseases. European Journal of Medicinal Chemistry. 110: 267-279.

García-Hansen, I. \& Gaviría-Chiquazuque, J.F. (1996). Estudio de los manglares de San Andres Isla: Extensión y distribución, estructura, productividad, degradación de hojas y otros análisis. Tesis profesional. Facultad Biología Marina, Universidad de Bogotá Jorge Tadeo Lozano.

García, Y., Rangel, J.O., Fernández, D. (2011). Palynological flora of the wetland from the aquatic, swamp and floodplain vegetation of the Córdoba and Cesar departments (Colombian Caribbean). Caldasia. 33 (2): 573-618.
Kumazawa, S., Murase, M., Momose, N., Fukumoto, S. (2014). Analysis of antioxidant prenylflavonoids in different parts of Macaranga tanarius, the plant origin of Okinawan propolis. Asian Pacific Journal of Tropical Medicine. 7 (1): 16-20.

Kuropatnicki, A., Szliszka, E., Krol, W. (2013). Historical aspects of propolis research in modern times. Evidence-Based Complementary and Alternative Medicine. 964149: 1-11.

Li, F., Awale, S., Zhang, H., Tezuka, Y., Esumi, H., Kadota, S. (2009). Chemical constituents of propolis from Myanmar and their preferential cytotoxicity against a human pancreatic cancer cell line. Journal of Natural Products. 72 (7): 1283-1287.

Lotti, C., Campo, M., Piccinelli, A.L., Cuesta-Rubio, O., Márquez, I., Rastrelli, L. (2010). Chemical constituents of red Mexican propolis. Journal of Agricultural and Food Chemistry. 58 (4): 2209-2213.

Lowy-Ceron, P. (2000). Flora vascular terrestre del archipielago de San Andrés y Providencia. Biota Colombia. 1 (1): 109-124.

Magalhães, F. \& Ribeiro, F, A. (2009). Pollen morphology of the shrub and arboreal of mangroves of Northeastern Brazil. Wetlands Ecology and Management. 17 (5): 423-443.

Matos, V.R. \& dos Santos, F. (2016). The pollen spectrum of the propolis of Apis mellifera L. (Apidae) from the Atlantic Rainforest of Bahia, Brazil. Palynology. 41 (2): 207-215.

Melliou, E., Stratis, E., Chinou, I. (2007). Volatile constituents of propolis from various regions of Greece-antimicrobial activity. Food Chemistry. 103 (2): 375-380.

Miguel, M., Nunes, S., Dandlen, S., Cavaco, A., Antunes, M. (2014). Phenols, flavonoids and antioxidant activity of aqueous and methanolic extracts of propolis (Apis mellifera L.) from Algarve, South Portugal. Food Science and Technology. 34 (1): 16-23.

Oliveira, L. \& Ribeiro, F.A. (2018). The presence of Fabaceae in the pollen profile of propolis produced in northesasern Brazil. Acta Botanica Brasilica. 32 (4): 602-614.

Olivieri, C., Silvestrin, C.; Gambato, G., Oliveira, M., Salvador, M., Moura, S., Ferreira, F., Kömmling, F., Collares, T., Borsuk, S., Dellagostin, O. A., Pêgas, J. A., Roech-Ely, M. (2013). Chemical characterization, antioxidant and cytotoxic of Brazilian red propolis. Food and Chemical Toxicology. 52: 137-142.

Omar, R.M., Ogoli, J., Gray, A.I., Ebiloma, G.U., Clements, C., Fearnley, J., Ebel, R.A., Zhang, T., De Koning, H.P., Watson, D.G. (2016). Chemical characterization of Nigerian red propolis and its biological activity against Trypanosoma brucei. Phytochemical Analysis. 27 (2): 107-115.

Palacios, R., Ludlow-Wiechers, B., Villanueva, R. (1991). Flora palinológica de la reserva de la Biosfera de Sian Ka'an Quintana Roo, México. Centro de Investigaciones de Quintana Roo. Seditorial Cioro. México. p. 351.

Piccinelli, A.L., Lotti, C., Campone, L., Cuesta-Rubio, O., Campo, M., Rastrelli, L. (2011). Cuban and Brazilian red propolis: botanical origin and comparative analysis by High-performance Liquid chromatography-photodiode array detection/electrospray ionization tandem mass spectrometry. Journal Agricultural and Food Chemistry. 59 (12): 6484-6491.

Rodríguez Y., Sánchez.Catalán, F., Rojano, B., Durango, D., Gil, J., Marín-Loaiza, J. (2012). Physicochemical characterization and evaluation of antioxidant activity of 
propolis collected in the Atlantic department, Colombia. Revista UDCA Actualidad \& Divulgación Científica. 15 (2): 303-311.

Salamanca, G. (2017). Origen, naturaleza, propiedades fisicoquímicas y valor terapéutico del propóleo. Ibagué. Sello Editorial de la Universidad del Tolima. p. 365.

Salamanca, G. \& Osorio, M.P. (2018). New Insular red propolis from Colombia: Botanical origin, Biological and Chemical markers. $30^{\text {th }}$ International Symposium on the Chemistry of Natural Products. Atens, Greece.

Salamanca, G., Osorio, M.P., Chávez, J, M., Ruiz, J., Torres, A.L. (2014). Valoración biométrica de poblaciones de abejas de Apis mellifera (Hymenoptera: Apidae) de San Andrés Isla, Colombia. Revista de la Asociacion Colombiana de Ciencias Biológicas. 26 (1): 296.

Salatino, A., Fernandes-Silva, C.C., Righi, A.A., Salatino, M.L. (2011). Propolis research and the chemistry of plant products. Natural products reports. 28 (5): 925-936.

Silva-Carvalho, R., Baltazar, F., Almeida-Aguiar, C. (2015). Propolis: A complex natural product with plethora of biological activities that can be explored for drug development. Evidence-Based Complementary and Alternative Medicine. ID 206439.

Sulaiman, G.M., Ad'hiah, A.H., Al-Sammarrae, K.W., Bagnati, R., Frapolli, R., Bello, E., Uboldi, S., Romano, M., Panini, N., Scanziani, E., Pezzolato, M., Erba, E., D'Incalci, M. (2012). Assessing the anti-tumour properties of Iraqi propolis in vitro and in vivo. Food and Chemical Toxicology. 50 (5): 1632-1641.

Theobald, W., Krahulik, J., Rolling, R. (1979). Trichome description and classification. Anatomy of the dicotyledons, I. Editorial C.R. Metcalf \& L. Chalk, Claredon Press, Oxford. 40-53.

Trusheva, B., Popova, M., Bankova, V., Simova, S., Marcucci, M.C., Miorin, P.L., da Rocha, F., Tsvetkova, I. (2006).
Bioactive constituents of brazilian red propolis. EvidenceBased Complementary and Alternative Medicine. 3 (2): 249-254.

Uribe, J. \& Urrego, L. (2009). Enviromental management of mangrove ecosystems: An approach for the Colombian case. Revista Gestión y Ambiente. 12 (2): 57-72.

Urushisaki, T., Takemura, T., Tazawa, S., Fukuoka, M., Hosokawa-Muto, J., Araki, Y., Kuwata, K. (2011). Caffeoylquinic Acids Are Major Constituents with Potent Anti-Influenza Effects in Brazilian Green Propolis Water Extract. Evidence-Based Complementary and Alternative Medicine. ID 252914.

Valente, M., Baltazar, A., Henrique, R., Estevinho, L., Carvalho, M. (2011). Biological activities of Portuguese propolis: Protection against free radical-induced erythrocyte damage and inhibition of human renal cancer cell growth in vitro. Food and Chemical Toxicology. 49: 86-92.

Valle, A, G., Osorno-Arango, A., Gil-Agudelo, D. (2011). Estructura y regeneración del bosque de manglar de la ciénaga de Cholón, Isla Barú, Parque Nacional Natural Corales del Rosario y San Bernardo, Caribe Colombiano. Boletín de Investigaciones Marinas y costeras. 40 (1): 115-130.

Velandia, M., Restrepo, S., Cubillos, P., Aponte, A., Silva, L.M. (2012). Catálogo fotográfico de especies de flora apícola en los departamentos de Cauca, Huila y Bolíva. Bogotá, Instituto Humboldt. Editorial Ediprint Ltda. p. 84.

Velasquez, C. \& Rangel, O. (1995). Atlas palinológico de la flora vascular del páramo I. Las familias más ricas en especies. Caldasia. 17 (82-85): 509-568.

Wagh, V. (2013). Propolis: A wonder bees product and its pharmacological potentials. Advances in Pharmacological Sciences. 308249: 1-11.

Willard, D., Cooper, S., Gámez, D., Jensen, J. (2004). Atlas of pollen and spores of the Florida everglades. Palynology. 28: $175-227$. 